\title{
Interview with John Rogers
}

\author{
John A Rogers*,1 \\ ${ }^{1}$ Departments of Materials Science \& Engineering, Biomedical Engineering \& Neurological Surgery, Northwestern University, \\ Evanston, IL USA \\ *Author for correspondence: jrogers@northwestern.edu \\ “Electronic technology has developed very rapidly in the consumer world and if this progress can \\ be harnessed and applied to problems in human healthcare then this area of bioelectronic \\ medicine could become a fruitful area of research. "
}

First draft submitted: 5 January 2018; Accepted for publication: 26 April 2018; Published online:

25 May 2018

\section{What led you into bioelectronic research?}

I came into the field in a roundabout way. My core expertise is in material science generally and electronic material science and electronic devices specifically. I got my first real start in that field as a member of the technical staff at Bell Laboratories. In those days in the early 1990s, we were working on unusual classes of electronic materials that enable construction of flexible electronic systems on thin sheets of plastic. We were looking into opportunities for use in applications such as paper-like displays, roll-up displays and very low cost radiofrequency identification tags. These types of devices benefit from the flexible mechanics and ability for low cost manufacturing by printing-type techniques.

For us at that time, this was an interesting area of material science with many potential applications. When I moved to the University of Illinois, I began to think more broadly and to plan a set of research directions in an academic environment. One consequence was that we launched efforts in a wider variety of constituent materials for similar and even more advanced platforms. We continued to be interested in displays and tags but we expanded to large-area, high-performance deployable electronics through programs funded by the department of defense.

I was giving talks on this research and one of these was at the University of Pennsylvania, where they have a very large medical school, hosted by the Department of Electrical Engineering. A few neuroscientists from the medical school happened to attend the talk, perhaps because the title/abstract looked interesting to them. After the presentation, they asked me whether it was possible to put these flexible electronics on the brain, to possibly measure electrical activity associated with neural function. This idea struck me as a really interesting, potentially an important direction, previously unexplored - exploiting electronics with flexible mechanics to wrap onto curvilinear surfaces of brain, or the body more generally, to integrate with biology in a more generic sense.

I think that this was probably the nucleating point for what has turned out to be a very productive area of research. The work involves using our expertise and knowledge of advanced electronic materials in integrated circuits, radios, wireless communication systems in consumer electronics, but for a new set of opportunities. We have also extended what one can think of as biocompatible electronics not only in terms of shapes and geometries but also at the level of the soft mechanics found in biology. These systems are much different that the sorts of rigid, planar computer chip that currently dominate consumer gadgetry, critically important for enabling high information content, conformal, noninvasive interface.

That biotic-abiotic interface becomes quite interesting from the standpoint of the fundamental science, a one moves away from consumer devices into biomedical systems. This transition was accelerated greatly by my students' enthusiasm. There's something more satisfying about working on scientific topics that have the potential for beneficial effects on human health than for a new display for a smart phone. I found that my students have been much more passionate around the healthcare-related platforms.

So I would say starting from zero in 2003, this biointegrated bio-electronics approach now represents more than two-thirds of what we do, and it is a very large group. We have many different activities in this space, and the vast majority has a tight interface to clinical needs. Just as our start in this field was launched by interest from neuroscientists, much that we now do has this important clinical connection. The open-ended discovery mode of 
science is great and we engage in that too but understanding the needs and interacting with people in a healthcare environment has been a very important part of what we do.

\section{Could you describe some of your work to design these thin ultrastretchable electronics?}

Our approach involves a combination of interdisciplinary-style research that spans several traditional fields of the study. Material science is at the core, as the materials are essential and enabling for these functional mechanically compliant platforms. At another level, it includes mechanical engineering and mechanical science as the materials must move and deform and also interface with soft tissues of the body. Chemistry is important part of the biosensors that we integrate into these systems and in understanding the reactions involved with insertion of these electronic systems into biological fluids. Basic physics also comes into play in terms of how to understand the biotic-abiotic interface, along with biology and bioengineering. Many different kinds of expertise come together for maximum impact. We can do this effectively because of the broad expertise that is embedded in the group. Another requirement is in a collaborative style, essential for the clinical interface but also in other ways to leverage our core competencies and to enable broader and higher impact outcomes.

In terms of nuts and bolts, we found that the geometrical attributes of materials in these systems are in many cases as important as the materials themselves and their atomic composition. A key insight, and a fundamental building block material in our systems, invovles nanoscale forms of silicon as a high performance semiconductor. Silicon is traditionally used in electronics as a wafer or plate of material on top of which circuit devices are constructed. We take those wafers and chemically remove from the near surface regions ultrathin nanoscale ribbons and membranes of silicon such that by virtue of their small thicknesses, they adopt highly flexible, even floppy, mechanics. Configuring these elements into wavy geometries, either with in-plane serpentine shapes or out-of-plane waves, and integrating them with a soft matrix polymer material yield a hard-soft composite with properties that can be engineered to provide mechanics precisely matched to those of the tissue at the target point of integration.

This overall strategy of engineered composite materials structures has worked well for us in rendering what are traditionally hard forms of technology into soft biocompatible systems. As you can see, it involves material science, mechanics and electrical engineering coming together as a set of concepts. An overarching rationale is, whenever possible, to try and maintain alignment, in terms of materials, manufacturing and device designs, with the consumer electronics industry. By doing so, it is possible to accelerate the maturation of the technology and to facilitate its move out of the academic lab as a curiosity and into something that is broadly impactful.

We develop new materials chemistries, but only when we have to; otherwise, we look for ways to use silicon in clever constructs such as the one I just described. By taking this strategy, we maintain a materials platform that is common with the 800-pound gorilla that is consumer electronics, such that we can leverage advances in that technology for our own purposes, in rapid ways. This has been a good strategy for us as we can exploit industry knowledge and conventional systems and modify them in ways that allow their use in completely different modes, for biointegration.

\section{Does this type of module have a potential application in bioelectronic medicine?}

We have looked at many points of integration. With soft electronics, there are many places where you can bring technology to bear on challenging disease states or targeted organ systems. Most of our work has focused on the heart, the brain and the skin, although we have also carried out other work on the bladder, peripheral nervous system and some on the spinal cord. In these systems, data collected in the context of bioelectronic medicine is extremely valuable as a feedback for the control of the therapeutic function of the device platform.

We have many active programs in sensors as noninvasive skin-mounted devices and as chronic implants, as well as bioresorpable devices. This latter area of technology is particularly exciting because it intersects strongly with the notion of a medical role for engineered systems. We typically carry out electrical or thermal stimulation as a therapeutic intervention. We also have programmable structures for microfluidic delivery, where drug release is an important possibility. By combining these kinds of actuation modalities with multimodal sensors, we can close the loop and operate in a feedback scheme that can enhance health and wellness and function of targeted organs.

We have done a lot on the bladder, where we are attempting to generate a set of technologies that allow for a nonpharmacological treatment of bladder dysfunction. A specific example is the development of a soft strain gauge that mounts around the bladder to measure the extent of filling. Based on that data, we can then either induce or prevent a voiding event by electrically interfacing to a pelvic nerve. This closed feedback loop approach 
is interesting, whereby we operate the devices in a way that allows their long-term survivability. In many cases, the devices must operate for the life of the patient $-10,20,30$ years or more, depending on the age of the patient.

Related to this is the idea of bioresorpable platforms that offer similar types of function, with appropriate materials and device construction that allows for their controlled bioresorption over time. In other words, these systems gradually disappear and eliminate themselves when no longer needed, thereby eliminating a requirement for a secondary surgical extraction. In this kind of physically transient design, the engineered system begins to look conceptuallly like a chemistry-based approach or a pharmacologically based approach to treating disease or facilitating wound healing.

In that context, we now have devices that can accelerate the regeneration of peripheral nerves through electrical stimulation. Through the processes of bioresorption, the devices then eliminate themselves after a few weeks, therefore providing continuous stimulation during the critical period of recovery and then naturally dissolving into the body fluids in a harmless manner. This type of operation avoids the need to perform a very delicate and risky surgery to remove the hardware from the fragile healed nerve.

Those are two examples of areas where some of these ideas in material science and device design are beginning to move toward embodiments that represent different areas of bioelectronics and medicinal value.

\section{Could you describe your work using capacitive coupling between tissue \& electronics for long-term electrophysiology?}

We have been working in this area for a long time. The very thin construction of these flexible devices enable mechanical flexibility, but it also creates very daunting challenges in encapsulation and in the creation of biofluid barriers. As you can imagine, devices that laminate onto the surface of the heart or brain and that are required to last a long time, the electronics must be encapsulated to prevent leakage currents from moving out into the surrounding tissue, first, and to prevent the penetration of biofluids into the active regions of the device where they can degrade the performance. Essentially, what is needed is a very thin but defect-free, perfect material layer that is impermeable to water and can be applied to the front and back sides of these thin sheets. It turns out that this is an extremely difficult materials engineering problem.

By analogy, consider the design approaches currently used for chronic long-term implants, such as pacemakers or deep brain stimulators. In these cases, all of the electronic components and the power supply systems are encapsulated in a titanium or ceramic can with walls that can be millimeters in thickness. This enclosure prevents biofluids from penetrating the device and entering the active electronics. With thin, flexible devices, this type of approach does not work because a few millimeters worth of titanium kills the mechanics and the kind of conformal integration thatis critical to the operation of these types of devices.

Last year, we published a solution to this challenge, as the outcome of work on this topic for nearly half a decade. The essential problem is that even a single, tiny pinhole can destroy the device as water will enter that pinhole and then propagate laterally across the whole system. One needs layers that are impermeable to water and are submicron in their thickness, completely free of defects over macroscopic areas, for example, tens of square centimeters. We have been able to create layers of this type by growing thin dense films of glass on surfaces of silicon wafers. As a growth process, these layers can be created at a very high levels of perfection, essentially zero defects over the surface of the wafer.

We also established a method for releasing these layers of glass from the wafer so they can be used to cap off the surfaces of our flexible electronic devices. As a result of their extreme thin geometry, they are flexible, resulting in a floppy glass material due to its thin geometry, capable of sealing the entire front size and back sides of the flexible electronic sheet with no surfaces exposed. This layer also works extremely well as a biofluid barrier. A nondirect coupling mechanism is then required to measure electrical activity, as there are no exposed metal electrodes. As a result of the thin geometry, changes in potential on the surface of the brain can be capacitively coupled to sensors located on the surface of the underside of the glass. This route achieves long-term, stable operation of the devices while immersed in biofluids.

\section{Your group developed this system for use in cardiac electrophysiology. What more needs to be done to develop biocompatible, flexible electronic implants?}

We have demonstrated measurements of electrophysiology across the surface of the heart, the epicardial surface. We also have unpublished data in which these same platforms have been adapted for monitoring brain function. Recording from the surface of the brain is mcch different than recording from the heart. It is easier because the 
brain does not move as much as the heart, but it is harder because the signal levels are much smaller. However, we have sufficient signal to noise with the same platform and we have long-term in vivo demonstrations showing stable operation for extended periods.

The disadvantage with the capacitive approach is that electrical stimulation is not very effective, so while the method is capable of sensing, it is difficult to stimulate by injecting current, which is an important part of bioelectronics medicine. We have been working on ways to embed local patches of conductive materials in the thin glass layer that can then electrically couple the surrounding tissues to the underlying active electronics without compromising biofluid permeation; that is, the barrier seal associated with the glass. It turns out that there are ways to do this and we are finishing up a manuscript that summarizes our approach to this second challenge.

I think that these two concepts, the conductive throughports and the perfect ultrathin glass layers, go a long way toward solving the interface challenges between flexible electronics and biological tissues, at least from the electrical standpoint. The next step is to integrate optoelectronic functionality, i.e. light emission and detection, and also the sampling of fluids or delivery of drugs. Ways of building multifunctionality into these platforms in a scalable way are needed so we move beyond an electrical mode of interaction to a multifunctional mode involving an exchange of chemistry, light or heat.

A further requirement is in scale up and deployment. The things we can do in an academic lab are sophisticated compared to anything out there but we still cannot produce full human brain-scale devices, for example. Our current devices are about the size of a postage stamp, as consequence of practical limitations of academic environments. It is hard to scale up without access to industrial level manufacturing capability but this is where we would like to go. For example, rather than mapping only a small region of the brain at high resolution, the whole brain could be mapped and as opposed to 10,000 measurement and stimulation sites there could be 10 million.

At one level this is an engineering scale up. There are, however, a lot of fundamental challenges that will have to be worked out within that scale up process. If we can do this, then these kinds of platforms can be made broadly available to the neuroscience community and many advances will follow from that. Understanding in neuroscience is often driven by the development of new tools and technology capabilities as much as by the development of new concepts. These types of technologies could therefore really drive progress and understanding.

Ultimately, progress can be made in technology but improved understanding of how the brain works is needed to provide positive feedback. As we come to understand better how the brain works we can design more relevant technologies and then those technologies will lead to deeper insights into brain function and you kind of go back and forth like that so I think it is a very exciting time. So my group is active and there are a number of other groups obviously doing some pretty interesting things as well, and I think that the broader field of brain science, bioelectronics medicine is poised for great progress in the coming years.

\section{How do you think the field of bioelectronic medicine will develop over the next 5-10 years?}

I am a little bit biased because as as an engineer I would like to believe that engineered systems such as bioelectronic medicines will have impact. I do, however, think that many people share that view, some of whom are not engineers. This is a very frontier area.

Electronic technology has developed very rapidly in the consumer world and if this progress can be harnessed and applied to problems in human healthcare then this area of bioelectronic medicine could become a fruitful area of research. It is probably overly optimistic to think that every disorder or disease state could be treated in this way, but it could be a very powerful compliment to a pharmaceutical approach. In many cases, bioelectronic medicines have less risk as they represent a more targeted approach. These engineering systems are employed in specific regions of the body, as opposed to drugs that permeate every part of the body in an indiscriminate way. We are excited about bioelectronic medicine and I think that there are a number of foundational ideas now available to the community that will help accelerate the development of the technology.

\section{Financial \& competing interests disclosure}

The author has no relevant affiliations or financial involvement with any organization or entity with a financial interest in or financial conflict with the subject matter or materials discussed in the manuscript. This includes employment, consultancies, honoraria, stock ownership or options, expert testimony, grants or patents received or pending, or royalties.

No writing assistance was utilized in the production of this manuscript. 


\section{Disclaimer}

The opinions expressed in this interview are those of the interviewee and do not necessarily reflect the views of Future Medicine Ltd. 